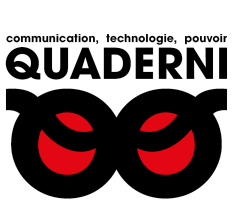

\title{
Quaderni
}

Communication, technologies, pouvoir

96 | Printemps 2018

Smart city : "fiction" et innovation stratégique

\section{De l'audace technique à la conformation politique? Quelques hypothèses de retour de la Silicon Valley}

\section{Stève Bernardin}

\section{(2) OpenEdition}

12 Journals

Édition électronique

URL : https://journals.openedition.org/quaderni/1176

DOI : 10.4000/quaderni.1176

ISSN : 2105-2956

Éditeur

Les éditions de la Maison des sciences de l'Homme

Édition imprimée

Date de publication : 15 mai 2018

Pagination : 43-57

\section{Référence électronique}

Stève Bernardin, « De l'audace technique à la conformation politique? Quelques hypothèses de retour de la Silicon Valley », Quaderni [En ligne], 96 | Printemps 2018, mis en ligne le 15 mai 2020, consulté le 28 septembre 2021. URL : http://journals.openedition.org/quaderni/1176 ; DOI : https://doi.org/ 10.4000/quaderni. 1176 


\section{$D$ ossier}

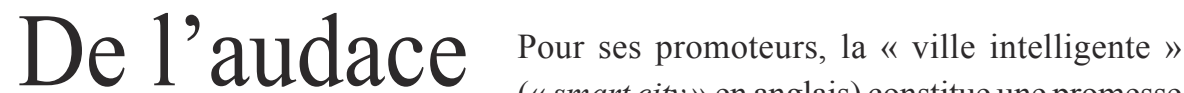
(«smart city» en anglais) constitue une promesse technique à la conformation politique? Quelques hypothèses de retour de la Silicon Valley indiscutable de progrès. Le développement des technologies numériques permettrait ainsi, par exemple, une optimisation de la gestion des ressources naturelles, dans nos lieux d'habitation comme de travail et de loisir, en autorisant un suivi en temps réel des consommations d'énergie. Il amènerait aussi à l'amélioration des conditions de circulation et de stationnement, en proposant une géolocalisation des zones d'encombrement ou de congestion, à partir de capteurs installés à même les chaussées et les trottoirs en ville. Chaque usager pourrait alors bénéficier d'un service à moindre coût, parfaitement adapté à ses besoins individuels. Il aurait en outre la possibilité de s'exprimer quant aux politiques urbaines, de gestion de l'eau ou des déchets par exemple, à partir d'applications téléchargées sur son téléphone portable.

La « ville intelligente », dans cette optique, constituerait une réponse idéale à tous les maux des villes contemporaines, en termes

Stève environnementaux, économiques et sociaux. Bernardin C'est en tout cas le discours porté par les scientifiques les plus technophiles, aux côtés des industriels du secteur numérique. Il n'est

LATTS pas sans détracteurs, tour à tour journalistes, essayistes ou philosophes ${ }^{1}$. Toute critique paraît néanmoins se heurter à un discours paré de la force de l'évidence.

Des travaux académiques s'inspirent de l'histoire des techniques pour analyser avec précaution ce qui apparaît comme un « idéal auto-réalisateur », dont la seule évocation suffirait à faire advenir toute la réalité politique et sociale ${ }^{2}$. À la suite 
d'Antoine Picon, ils invitent à rester prudents quant au discours en vogue sur la «ville intelligente ». Des thèses de doctorat s'inscrivent dans une même filiation en s'intéressant aux transformations concrètes associées au développement des technologies numériques au sein des villes françaises ou européennes ${ }^{3}$. Ces recherches laissent de côté le discours porté par les industriels du secteur numérique, pour mettre l'accent sur les pratiques observées au niveau des municipalités et des intercommunalités.

Notre approche est différente. Empruntant à une démarche de sociologie des problèmes publics, elle invite à focaliser notre attention sur l'argumentaire des industriels en faveur de la « ville intelligente », ou plutôt sur les conditions politiques et sociales de sa production et de sa mise en circulation ${ }^{4}$. Elle amène à nous intéresser d'abord aux porteurs de cause : qui sont-ils précisément, et comment en viennent-ils à prendre la parole en public ? Elle passe ensuite par l'approfondissement des formes prises par leur mobilisation : reposent-elles sur une forme de spécialisation, voire de division de travail entre industriels et scientifiques, par exemple, ralliés en vue de crédibiliser et de légitimer les propositions avancées ? En cherchant à le comprendre, nous tenons à objectiver les ressources dont disposent ces acteurs pour porter leur cause en public, autant que les contraintes qui sont les leurs pour ce faire, au-delà de tout présupposé d'omnipotence a priori de leurs porte-paroles ${ }^{5}$.

Notre analyse se fonde en priorité sur un matériau réuni lors d'une enquête de terrain menée aux États-Unis, dans le cadre d'une recherche collective plus vaste, conduite aux côtés de quatre chercheurs du Centre de Sociologie de l'Innovation et d'une douzaine d'étudiants de l'option «Affaires Publiques et Innovation » de l'École Nationale Supérieure des Mines de Paris, du 7 au 20 février 2016 en Californie ${ }^{6}$. Pour le présent article, nous disposons plus spécifiquement de séquences d'observation ethnographique de réunions auxquelles nous avons pu assister au plus près des quartiers généraux de l'industrie du secteur numérique, au cœur de la Silicon Valley. Nous mobilisons aussi une dizaine d'entretiens approfondis avec des dirigeants d'entreprises, ainsi qu'avec un groupe restreint de leurs interlocuteurs universitaires et institutionnels à San Francisco.

\section{« Il faut penser emploi, pas technologie » : une euphémisation de l'argument technique en petit comité}

Il n'est pas simple d'enquêter sur l'industrie numérique. Depuis la France, déjà, nos demandes d'entretiens restent pour la plupart sans suite. Un courriel apparaît toutefois comme le sésame tant attendu, quelques jours avant le départ pour la Californie. Il nous invite à faire connaissance avec son auteur en marge d'une conférence de l'« International Society of Service Innovation Professionals »(ISSIP), à laquelle nous sommes conviés au cœur de la Silicon Valley. Notre interlocuteur, directeur des programmes universitaires de la compagnie IBM, est par ailleurs secrétaire et trésorier de l'ISSIP, une association fondée en 2012 par les responsables de trois entreprises majeures du secteur, Cisco, IBM et Hewlett-Packard, rejointes depuis lors par de nombreuses autres firmes, à l'image de Google, Intel, Oracle ou encore Samsung ${ }^{7}$. Jim 
Spohrer nous donne peu d'informations sur la conférence à laquelle il nous invite, même s'il nous laisse entendre qu'il nous sera possible d'y rencontrer les spécialistes les plus qualifiés pour nous parler de « villes intelligentes ».

Une fois l'invitation acceptée, nous nous préparons à assister à une grande conférence abordant des thèmes essentiellement techniques, en relation avec le développement des technologies numériques. Tout nous laisse en effet penser qu'il sera question d'innovation avant tout, en présence d'un grand nombre de spécialistes du sujet. La trajectoire de Jim Spohrer, tout d'abord, est celle d'un scientifique assumant ses compétences techniques, diplômé du MIT et de Yale avec une thèse en informatique ( Computer science "), aujourd'hui "ingénieur de haut niveau » de la compagnie IBM (« distinguished engineer »). Il en va de même de la présidente de l'ISSIP, Monique Morrow, travaillant depuis une quinzaine d'années pour une autre grande compagnie du secteur, Cisco. L'audience de la conférence promet par ailleurs d'être importante, puisque l'association compte près d'un millier d'adhérents, entrepreneurs ou scientifiques pour la plupart.

Le décor semble idéal pour la rencontre, puisque nous découvrons un lieu de rendez-vous en plein cœur de la Silicon Valley. Plus précisément, la conférence a lieu dans un cadre universitaire, au sein de l'antenne locale de Northeastern University. Ses locaux jouxtent ceux d'une grande compagnie du secteur informatique. Ils permettent d'envisager des présentations en amphithéâtre, suivies d'ateliers en groupes restreints, comme c'est d'ordinaire le cas, du moins lors de rencontres similaires auxquelles nous avons pu assister en France. À l'horaire indiqué, nous commençons pourtant à en douter. Les organisateurs nous invitent en effet à les rejoindre dans une petite salle de cours, où nous accueillons moins d'une vingtaine de présents, auxquels s'ajoutent quelques participants en visioconférence. Le tour de table, formel mais décontracté, laisse entrevoir un milieu d'interconnaissance entre responsables industriels et universitaires locaux, pour la plupart ingénieurs de formation. À ce stade, même en petit comité, nous imaginons toujours un débat essentiellement technique, peut-être pour convenir des dernières innovations, entre spécialistes des « villes intelligentes ».

Le programme du jour s'organise autour de la présentation d'un intervenant principal, P. K. Agarwal. Jim Spohrer le présente comme un ingénieur de talent, formé en Inde, à l'Indian Institute of Technology de Delhi, puis aux États-Unis, à l'Université d'État de Californie à Berkeley. Il précise qu'il occupe actuellement des fonctions de doyen et de responsable administratif ( Chief Executive Officer ») de l'antenne de Northeastern University dans la Silicon Valley. Cette présentation nous conforte dans l'attente d'un discours essentiellement technique de la part de l'universitaire. Dès ses premiers mots, toutefois, il n'est pas question de capteurs ou d'applications numériques, ni même d'innovations ou de standards technologiques. L'intervenant privilégie plutôt une mise en perspective historique de ce qu'il considère comme de grandes mutations en cours, aussi profondes et durables que celles ayant vu émerger la révolution industrielle ou l'arrivée de 
l'automobile en ville, au début du siècle. Pour lui, le développement des technologies numériques serait à envisager sur le long terme, comme une promesse de rupture allant bien au-delà du seul périmètre scientifique et technique.

L'universitaire invite à raisonner par analogie en exposant la cas du moteur à combustion. $\mathrm{Si}$ son développement, aux débuts de l'automobile, a remis en cause le travail de milliers de conducteurs de calèches, qu'en sera-t-il de l'avènement annoncé des véhicules autonomes, par exemple vis-à-vis des conducteurs de bus ou de taxis? "Ce que je vous dis n'est pas neutre. Il faut qu'on change de logiciel pour parler du numérique. Il faut penser emploi, pas technologie ${ }^{8}$. » L'affirmation se veut péremptoire. P. K. Agarwal ajoute : "On a tendance à surestimer l'impact des grands changements sur le court terme, mais aussi à le sous-estimer sur le long terme. » Ce faisant, l'universitaire met en avant le besoin d'une formation adaptée aux mutations en cours et à venir d'un marché de l'emploi qu'il considère comme nécessairement changeant. Il égrène une liste des métiers qu'il considère comme « en danger » (notamment postier, géomètre ou fermier), avant d'insister sur l'importance de construire de nouveaux "partenariats entre industrie, université et gouvernement", en vue de "former à nouveau les travailleurs [re-skilling] et les former à un plus haut niveau de qualification [up-skilling]. »

L'intervenant parait ici bien dans son rôle de responsable d'un pôle universitaire cherchant à développer des activités de formation continue à destination des salariés de compagnies installées dans la Silicon Valley. Il poursuit en soulignant d'abord l'importance de «ne pas avoir peur de prendre des risques », dans une rhétorique libérale assumée, avant de faire l'éloge des start-ups du secteur numérique. Il en achève sa présentation en insistant sur les vertus de l'entreprenariat, avec un transparent affichant un Superman au torse barré d'un E, celui de «l'Entrepreneur ». P. K. Agarwal évoque aussi le soutien nécessaire des élus, "à même de comprendre l'intérêt d'anticiper le futur. " Il témoigne ainsi d'une initiative impliquant le maire de San José, ville la plus peuplée de la Silicon Valley, ayant permis la formation en six mois de près de mille travailleurs (pour la plupart jeunes adultes sans diplôme de l'enseignement supérieur, chômeurs de longue durée, ou vétérans de guerre), en partenariat avec de nombreuses compagnies locales, telles que Cisco, Google, Ebay, LinkedIn ou IBM ${ }^{9}$.

L'accent mis sur l'emploi, et finalement sur des enjeux politiques, se comprend alors que s'ouvre le débat suivant la présentation de $\mathrm{P}$. K. Agarwal. L'intervenant, invité à revenir sur sa trajectoire professionnelle, révèle en effet un épisode important de sa carrière, quand il occupait des fonctions de responsable des politiques technologiques (« Chief Technology Officer ») auprès du Gouverneur de l'État de Californie, Arnold Schwarzenegger, pendant plus de cinq ans, de 2005 à 2010. Il témoigne ainsi de l'importance non seulement du soutien, mais aussi de l'attention des représentants politiques de l'État comme des municipalités en faveur du développement de l'emploi des populations locales. Son analyse rejoint alors celle de spécialistes universitaires de l'économie régionale de la Californie ${ }^{10}$. La rencontre de l'ISSIP permet ainsi de souligner l'importance 
d'un dialogue avec des responsables politiques attentifs au thème de l'emploi, plus encore qu'à celui de l'innovation technologique, ce dont témoigne en souriant Jim Spohrer : "C'est l'agenda secret de l'ISSIP d'être le syndicat des travailleurs [labor union] de l'industrie $d u$ service ${ }^{11}$. »

\section{L'objectivation d'une contrainte latente : la résistance des bureaucrates entre défiance et indifférence}

Une fois la conférence achevée, et face à nos demandes de précisions, Jim Spohrer nous accorde un long entretien. Notre interlocuteur met rapidement en avant la notion de « science du service » (« service science »), en référence au titre même de l'ISSIP, l'International Society of « Service » Innovation Professionals. Il y voit un projet de réponse à la complexité croissante du monde actuel, qu'il juge de plus en plus interdépendant et interconnecté. Dans le sens de P. K. Agarwal, il insiste sur ce qu'il considère comme une profonde mutation en cours du marché du travail, qui amènerait à s'interroger sur les compétences requises pour développer les métiers du futur, ne recouvrant plus les divisions sectorielles antérieures, en matière de transports, ou de gestion des déchets et de l'eau, par exemple. Jim Spohrer évoque l'existence d'une communauté scientifique traitant du sujet, par le biais de conférences et de revues comme « Service Science », créée en 2009 pour agréger des réflexions pluridisciplinaires sur ce thème, des mathématiques appliquées aux sciences de gestion.

Il justifie dans ce sens l'invitation de P. K.
Agarwal à l'ISSIP, en vue d'approfondir la connaissance collective du type de « services » à développer à l'avenir, en relation avec un ensemble de formations adaptées, aux côtés d'universités partenaires. Il n'insiste pas explicitement sur un objectif peut-être moins évident, en apparence, celui de contribuer à l'acculturation d'acteurs non seulement universitaires, mais aussi politiques, aux travaux de l'ISSIP. Jim Spohrer travaille pourtant à la reconnaissance d'un savoir formel contribuant à rationaliser et à théoriser le développement de nouveaux services à destination des élus. La tentative de structuration d'un sous-champ scientifique, celui de la «science du service », est importante à ce titre. Les « professionnels » de l'ISSIP, grâce à sa reconnaissance politique, pourraient en effet revendiquer un périmètre de compétence nouveau, légitimé par un ensemble de connaissances et de savoir-faire clairement identifiés et mis en scène, dans une logique bien connue des sociologues du travail et des professions ${ }^{12}$. Notre interlocuteur ne cache pas les enjeux économiques de la démarche. Il évoque une « science du service » visant aussi et surtout, « au-delà de questions purement académiques », à « trouver les nouvelles opportunités de création de valeur" en vue de "créer de nouveaux modèles économiques [business models] ${ }^{13}$. »

Quand nous l'invitons à préciser sa pensée, Jim Spohrer revient sur une genèse essentiellement économique de ses travaux sur le sujet, au sein de la compagnie qui l'emploie, IBM. Il évoque une crise importante de l'entreprise, à la fin des années 1990. Les ventes d'ordinateurs et de logiciels sont en baisse rapide, du fait notamment de politiques agressives de concurrents comme 
Apple ou Microsoft. Les dirigeants de la compagnie choisissent dès lors de recentrer leurs activités sur des missions de service. Jim Spohrer se remémore des débats avec les responsables commerciaux, privilégiant le concept de «Smarter Planet» à celui de « Smarter Systems », pour labelliser l'initiative en cours. Un dirigeant de la branche française d'IBM, en activité au sein de la compagnie depuis le milieu des années 1980, confirme une évolution en profondeur des stratégies commerciales de la firme, amenant à passer de services relativement standardisés, des années 1995 et 2000, à des solutions plus spécifiquement adaptées aux besoins des clients potentiels, autour de 2004 à $2005^{14}$. La mutation s'accompagne d'une réorganisation interne d'IBM, ainsi que d'un investissement à hauteur de cinq à dix milliards d'euros dans le rachat d'entreprises spécialisées dans la création de logiciels.

Comme le note le géographe Donald McNeill, en analysant les publications de la firme au cours de la dernière décennie, la stratégie commerciale de la compagnie se focalise rapidement sur la fourniture de services aux villes, plutôt qu'aux États, ou à d'autres compagnies privées. Les analystes de l'entreprise, en 2012, prévoient en effet le développement d'un marché de plus de cinquante-sept milliards de dollars dans ce domaine, pour la seule année $2014^{15}$. La proposition de services en matière de consommation d'énergie et d'offre de transports apparaît comme une priorité, à destination de villes de tailles non seulement importantes, mais aussi plus réduites. Le lancement du « Smarter Cities Challenge » vise initialement à mettre en lumière d'importants gains en efficacité en matière de gestion des politiques urbaines, pour les collectivités choisissant d'en appeler aux spécialistes d'IBM ${ }^{16}$. Entre 2011 et 2013, il prévoit l'envoi annuel, à titre gracieux, d'employés de la compagnie dans une centaine de villes candidates. L'initiative n'est pas uniquement philanthropique. Elle permet aussi aux experts de s'acculturer aux pratiques des administrations locales en vue du développement de futurs marchés.

Peter Williams, un collègue de Jim Spohrer, fait partie des équipes envoyées dans des villes lauréates du challenge proposé par IBM. En 2012, il se rend ainsi à Da Nang, au Vietnam, dans une localité de près d'un million d'habitants, en forte croissance démographique, située à 600 kilomètres de Hanoï et de Hô-Chi-MinhVille. Lorsque nous lui demandons de décrire son quotidien sur place, Peter Williams évoque un mois d'expérimentations concrètes auprès des autorités locales, avec une équipe réduite d'une petite dizaine d'experts d'IBM. Il insiste sur le temps passé à formuler un diagnostic des problèmes rencontrés à Da Nang. Il souligne une élévation du niveau de vie des habitants, s'accompagnant d'une augmentation importante du nombre d'automobiles en circulation ainsi que de la congestion urbaine en résultant. Notre interlocuteur en fait un problème de santé publique, en ajoutant que les denrées périssables, poissons ou fruits de mer notamment, tendent à se détériorer en l'absence d'une quantité suffisante de camions réfrigérés pour les transporter. Les hôpitaux de la ville observent au même moment une recrudescence des cas d'empoisonnement alimentaire. Peter Williams en arrive ainsi à un projet d'installation d'un système intégré 
de gestion informatique de trafic, avec l'aval du maire de la ville. Cet exemple lui permet d'insister face à nous sur l'importance d'un décloisonnement des logiques traditionnelles de l'action publique en ville, avant tout sectorielles, dans le sens de la démarche prônée à l'ISSIP ${ }^{17}$.

Notre interlocuteur évoque une approche similaire dans le cas de villes européennes ou américaines, comme San Francisco. Il s'avère toutefois bien moins précis lorsqu'il est question d'aborder le détail des projets menés en relation avec la collectivité. Nous avons l'occasion d'approfondir le sujet avec Krista Canellakis, l'adjointe au responsable de l'innovation ("Deputy Innovation Officer ») de la ville de San Francisco. Diplômée en urbanisme de l'University College London, elle n'est pas ingénieure de formation, à la différence de ses interlocuteurs d'IBM. Elle porte en outre son attention sur les petites entreprises, plutôt que sur de grandes compagnies, après une expérience personnelle dans la communication de projet au sein d'une start-up environnementale de la ville, 3Degress. Dès le début de l'entretien qu'elle nous accorde, l'accent est mis sur un problème d'adéquation entre l'offre commerciale et la demande municipale : "Il y a des choses nouvelles tous les jours dans les start-ups, mais elles ne sont pas toujours conçues dans une perspective de résolution de problèmes [problem-solving] ${ }^{18}$. » Krista Canellakis précise son propos en soulignant une méconnaissance des enjeux propres à l'administration des villes pour de nombreux acteurs économiques du secteur : « ces gens n'ont pas conscience des problèmes auxquels fait face la ville ${ }^{19}$. »

Dans la suite de l'entretien, notre interlocutrice confirme l'analyse d'un géographe, Alan Wiig, notant des problèmes importants de délais de livraison des logiciels et des applications mobiles, qu'il analyse comme autant d'indices d'une convergence difficile entre les priorités des bureaucrates municipaux et celles de leurs interlocuteurs commerciaux, comme dans le cas d'expérimentations similaires, menées par exemple par une équipe d'IBM à Philadelphie, en Pennsylvanie ${ }^{20}$. Jim Spohrer témoigne lui aussi d'un dialogue délicat à mettre en place, lorsqu'il insiste sur " la nécessité d'aller contre les routines » ou de "récompenser la prise de risques $^{21}$. » Peter Williams se fait plus explicite encore dans un texte dénonçant un cloisonnement des politiques municipales qui renverrait à des organigrammes rigidifiés, où chacun serait en lutte pour préserver son périmètre professionnel, au détriment de toute possibilité de réforme réelle ${ }^{22}$. Incidemment, il révèle ici une limite à l'exercice de la démonstration technique proposé à Da Nang ${ }^{23}$. Des groupes de bureaucrates peuvent en effet s'opposer fermement, ou se montrer indifférents, aux propositions des experts de compagnies privées, en arguant de spécialisations sectorielles développées depuis des décennies, dans les domaines des transports, des politiques de l'eau ou de l'énergie par exemple.

\section{Après Songdo et Masdar : une spécialisation du discours à destination des élus «à l'écoute »}

Les limites du discours techniciste des experts apparaissent clairement lorsqu'il est question d'évoquer les cas emblématiques de Songdo ou de Masdar. Le premier d'entre eux renvoie à un territoire de 600 hectares développé avec des spécialistes de Cisco à une soixantaine 
de kilomètres de Séoul, en Corée du Sud. La création de Songdo (ou « New Songdo ») répond à l'objectif initial d'attirer des investisseurs étrangers à proximité de l'aéroport international de la capitale, sur un site désigné comme une zone franche par les autorités étatiques. Près de trentecinq milliards de dollars d'investissements visent ainsi à doter la ville de grands espaces verts et d'une technologie de pointe, permettant à chaque habitant d'adapter sa consommation d'énergie à son estimation en temps réel. Après une dizaine d'années, cependant, moins de la moitié des 250000 habitants attendus ont emménagé. Le bilan paraît encore plus critique à Masdar (ou « Masdar City »), sortie de terre à une trentaine de kilomètres de la capitale d'Abou Dhabi, dans les Émirats Arabes Unis. Le projet vise initialement à témoigner de l'investissement des autorités locales dans les énergies renouvelables et l'efficacité environnementale, en prévision de l'épuisement annoncé des énergies fossiles, gaz et pétrole. Malgré quinze milliards de dollars investis, cependant, la ville n'accueille que quelques centaines d'habitants, au lieu des 50000 attendus.

«Je ne suis pas sûr de vouloir y vivre moimême ! ${ }^{24} \gg$ Peter Williams développe sa pensée en reconnaissant d'importantes avancées technologiques, dans « ces villes où tout est très efficace et bien vert», en insistant en même temps sur leur côté «top-down très technocratique ». Jim Spohrer va jusqu'à une forme de mea culpa : " on sait faire beaucoup de choses très intelligentes, mais on ne sait pas faire une ville entière $^{25}$. » Il précise en reprenant l'exemple de localités chinoises ou sud-coréennes : «Des gens peuvent y vivre, mais peut-être pas ceux qui ont vraiment le choix. " L'affirmation ne traduit pas uniquement l'endogénéisation d'une possibilité de critique technocratique à l'endroit des industriels du secteur numérique. Elle permet aussi et surtout de préparer une dénonciation possible de bureaucrates municipaux présentés comme insensibles à la prise en compte des besoins des habitants ou des usagers des services publics. Jim Spohrer revient ainsi sur la nécessité d'une « science du service » faisant la part belle aux analyses d'anthropologues, notamment, pouvant aider à concevoir une génération nouvelle de villes intelligentes, désormais adaptée aux besoins réels des citadins, ainsi qu'à leurs nouvelles conditions de vie avec les technologies numériques.

L'ingénieur d'IBM insiste en particulier sur des individus bientôt indissociables de leurs « assistants cognitifs », téléphones et autres objets connectés, en mesure d'enregistrer l'ensemble de nos rencontres ou de nos échanges présents, passés et futurs, « qui nous connaîtront mieux que nous-mêmes » et «qui pourront tout analyser pour nous dire ce qui doit être prioritaire. » Dans cette perspective, le marché de l'emploi ne sera pas le seul impacté par le développement des technologies numériques. Chaque citadin aura la possibilité d'expérimenter une vie radicalement nouvelle, au côté de ses « coaches personnels intégrés ${ }^{26}$. » Dans ce sens, les industriels de la Silicon Valley investissent non seulement dans la compréhension, mais aussi dans l'accompagnement de l'évolution qu'ils décrivent. Ils affichent en effet une forme d'altruisme visant à l'épanouissement de chaque individu, sans oublier pour autant le développement de produits et de services pouvant 
en découler, comme en témoignent les travaux de Marc Abèles ${ }^{27}$.

Dans notre cas d'étude, la démarche n'est pas sans usage politique. Les industriels défendent en effet un projet de citoyenneté renouvelée, passant par un accès facilité aux pratiques des responsables institutionnels. Peter Williams le confirme : "l'idée est de fournir les données qui pourront stimuler une discussion sur ce que fait le gouvernement ${ }^{28}$. " Il dépeint alors une évolution de l'argumentaire déployé par les industriels du secteur numérique sur le thème des villes intelligentes : " "obtenir plus de données", c'était pour les spécialistes de technologie, "faire plus avec moins", c'était pour les financiers, et "s'impliquer face au politique" [to engage with government], c'est pour le grand public. » L'usager des services publics devient ainsi un citoyen à part entière, en mesure d'exprimer satisfaction autant que mécontentement vis-àvis des responsables institutionnels. Philippe Sajhau, vice-président d'IBM France, ne dit pas autre chose en 2016 : «Les utilisateurs ont changé et pris le pouvoir, et, quand on parle d'utilisateur dans la ville, on parle de citoyen ${ }^{29}$. Dès lors, l'accent ne porte plus sur l'innovation technologique, mais sur la prise en compte de figures nouvelles de l'habitant ou de l'usager des services publics, à même d'exister et de se faire entendre en politique.

L'invitation des industriels à " écouter les citoyens » n'est pas neutre pour l'administration des villes. Comme le note le sociologue François Ménard, elle s'accompagne d'un présupposé tacite, celui d'une évolution en profondeur des pratiques bureaucratiques, en vue de « capter, ordonner et redistribuer les données nécessaires à l'efficacité et au développement de ces services $^{30}$. » La démarche paraît d'autant plus incontournable qu'elle est avancée au nom de la transparence des administrations concernées. Pour Philippe Sajhau, «la démarche dépasse les fonctions ordinaires de gestion de la mairie », par exemple en matière de gestion des transports ou des déchets, « en incluant une relation nouvelle avec les citoyens ${ }^{31}$. " L'argumentaire s'avère dès lors politique : il rappelle aux bureaucrates, potentiellement réticents à l'arrivée du numérique en ville, qu'ils travaillent pour des élus attentifs à la satisfaction de leurs administrés quant à la qualité du service proposé. Il permet aussi, en retour, de signifier aux maires qu'ils ont tout intérêt à suivre les préconisations des experts du domaine, en vue de satisfaire des citoyens soucieux de la transparence des administrations dont ils ont la responsabilité.

La rhétorique n'est pas portée uniquement par des ingénieurs de formation, comme Jim Spohrer ou Philippe Sajhau. Elle émerge aussi et surtout de spécialistes issus de grands cabinets de conseil. Peter Williams, bien que disposant au sein d'IBM du titre d'« ingénieur de haut niveau » (« distinguished engineer »), étudie ainsi la science politique avant d'obtenir une thèse de doctorat de l'École de Management de l'Université de Bath, en 1986. Il travaille ensuite comme " lobbyiste politique ", puis comme consultant en management au sein du cabinet PricewaterhouseCoopers, que rachètent les investisseurs d'IBM en 2002. L'observation n'a rien d'anecdotique : elle témoigne des ressources importantes déployées par les industriels du secteur numérique pour comprendre les règles 
du jeu politique local, en vue de s'y conformer, au moment même où leurs dirigeants initient leurs programmes de développement des « villes intelligentes ». Dans le cas de San Francisco, par exemple, l'enjeu retenu consiste bel et bien à convaincre les élus, en cherchant «à s'intéresser en priorité aux retours des citoyens quant à l'administration, à voir ce que chacun pense d'elle . $^{32}$ »

Le projet n'est pas sans référence au mythe d'une culture politique particulièrement ouverte au débat et à l'échange d'idées, ainsi qu'à la prise en compte des initiatives citoyennes dans la gestion au quotidien de la ville californienne ${ }^{33}$. Il fait par ailleurs écho à des priorités de campagne du maire démocrate de San Francisco, Edwin M. Lee, élu en 2011 et réélu en 2015. Né aux États-Unis de parents chinois, le diplômé en droit de l'Université de Berkeley met volontiers en avant son attention aux droits des migrants et des plus démunis, ainsi qu'à leur intégration aux activités économiques et culturelles de la ville. Il s'empare de la rhétorique de la participation des citoyens et de "l'inclusion digitale » (visant à lutter contre les inégalités d'accès aux technologies numériques) en créant en 2012 le « service de l'innovation civique » (le « Mayor's Office of Civic Innovation »). Directement rattaché au cabinet du maire, le service compte à peine une demi-douzaine d'employés. Il développe néanmoins un important programme d'actions visant à valoriser le développement des technologies numériques, pour « connecter la ville aux citoyens ${ }^{34}$. »

Le projet se traduit non seulement par une écoute nouvelle des demandes existantes, mais aussi par l'ambition affichée d'une prise de parole des habitants les plus pauvres de la ville, par le biais d'un accès facilité aux dispositifs numériques. Il comprend un vaste plan d'équipement des personnes âgées et des enfants des milieux considérés comme défavorisés, passant par la mise à disposition d'ordinateurs et d'applications dédiées à l'apprentissage du maniement de l'outil informatique. Le programme de la ville, porté par le maire en personne, reprend et développe ainsi les priorités fixées par les industriels du secteur numérique, dans le sens d'un argumentaire avant tout social, plutôt que technologique ou financier. L'évolution se double d'un changement plus discret, dans le profil même des bureaucrates recrutés à San Francisco. En 2012, le maire nomme ainsi un ancien consultant du cabinet PricewaterhouseCoopers, à l'image de Peter Williams, à la tête du service de l'innovation civique récemment créé, en qualité de « responsable des politiques d'innovation » («Chief Innovation Officer »). Les résistances bureaucratiques au projet de l'ISSIP semblent alors en passe d'être levées, au moins dans la ville de San Francisco.

\section{En guise de conclusion : apports et limites de l'hypothèse de la conformation politique}

Il serait présomptueux d'avancer des résultats de recherche définitifs à l'issue d'une enquête essentiellement exploratoire. Des pistes d'analyse nous paraissent toutefois intéressantes à mettre en débat. Elles invitent à remettre en question des présupposés tenaces quant à l'avènement annoncé des « villes intelligentes ». Une première piste renvoie au type d'argumentaire déployé par les industriels du secteur numérique. Contre toute 
attente, l'enquête ne permet pas d'insister sur le développement d'une rhétorique technique, invitant à célébrer les grandes innovations associées aux technologiques numériques. L'argument financier ne paraît pas non plus s'imposer. Au cœur de la Silicon Valley, en effet, une rhétorique différente semble se développer. Elle pourrait témoigner d'une capacité inattendue de repositionnement des industriels, face à la résistance discrète de bureaucrates réticents à leurs propositions de nouveaux services urbains. La mise en avant d'une demande sociale, au nom d'électeurs désormais interconnectés, s'imposerait alors comme une manière détournée d'en appeler aux responsables politiques des municipalités, pour les inviter à travailler avec des interlocuteurs extérieurs à l'administration, en mesure de répondre à des besoins explicites des citoyens. À l'encontre du présupposé répandu de toute puissance des industriels, l'analyse mettrait ainsi en lumière un travail de conformation des industriels à ce qu'ils identifient comme des règles du jeu politique local.

Au-delà même du cas des « villes intelligentes », l'investigation invite à prêter attention à des organismes comme l'ISSIP pour l'analyse des formes publiques de mobilisation des élites économiques. L'association, en effet, est loin de constituer une simple vitrine de l'innovation technologique. À la suite de Jim Spohrer, ses membres travaillent à en faire un lieu d'échange d'expériences entre industriels du secteur numérique, revenant ici volontiers sur des expérimentations jugées parfois décevantes, comme à Songdo ou Masdar. Ils participent ainsi, chemin faisant, à se doter de références communes, partagées et interprétées dans une même logique, en vue de porter leurs demandes en public. Le développement d'une « science du service », portée et diffusée lors des rencontres de l'ISSIP, vise plus précisément à légitimer l'approche des industriels en la parant des attributs d'autorité d'un savoir formel, conférant à ses détenteurs une crédibilité savante inédite. L'invitation de P. K. Agarwal, dans ce sens, contribue à nourrir la réflexion, tout en participant en retour à son acculturation aux priorités de l'ISSIP. Pas à pas, des pistes nouvelles sont ainsi explorées et affinées, en faveur d'arguments non plus techniques ou financiers, mais sociaux et politiques. De nouveaux entrants participent au processus de spécialisation en question, à l'image de Peter Williams. Le mettre en lumière, à travers un organisme comme l'ISSIP, invite à souligner l'ampleur des ressources à disposition des industriels en vue de porter leurs demandes en public.

En contre point, l'hypothèse d'une conformation politique ouvre le débat quant au rôle des élus face à leurs interlocuteurs économiques. D’ordinaire mésestimés par les sociologues des problèmes publics, ils semblent bel et bien constituer, au moins dans notre cas d'étude, un point de passage obligé pour les industriels. L'observation conforte au passage l'analyse plus générale d'élites économiques ne pouvant apparemment pas se passer du soutien de leurs interlocuteurs politiques, quand bien même leurs porte-paroles prônent un libéralisme avancé. Elle mériterait toutefois d'être testée et approfondie au prisme de nouvelles enquêtes de terrain. Les élus, en effet, sont loin de tous occuper des positions identiques au sein de l'espace politique. Les uns pourraient ainsi être en mesure de contourner 
ou de détourner les propositions des industriels, quand les autres n'auraient pas d'autre choix que de les valider. Le mettre en lumière permettrait de proposer une analyse plus précise des formes prises localement par les mobilisations publiques en faveur de l'avènement de la « ville intelligente », au-delà du cas de San Francisco. Il serait ainsi possible d'identifier des limites probables au processus de conformation des élites économiques aux règles du jeu politique, traduisant en fait une capacité différentielle des élus à imposer leurs propres priorités à leurs nouveaux interlocuteurs du secteur numérique.
$\mathrm{N} \cdot \mathrm{O} \cdot \mathrm{T} \cdot \mathrm{E} \cdot \mathrm{S}$

1. Par exemple Éric Sadin, La Silicolonisation $d u$ Monde. L'irrésistible expansion du libéralisme numérique, Montreuil, Éditions L'Échappée, 2016 ; Philippe Vion-Dury, La nouvelle servitude volontaire. Enquête sur le projet politique de la Silicon Valley, FYP Éditions, 2016.

2. Antoine Picon, Smart Cities. Théorie et critique d'un idéal auto-réalisateur, Éditions B2, 2013.

3 . Voir notamment la thèse récente d'Antoine Courmont (Politiques des données urbaines : ce que l'open data fait au gouvernement urbain, IEP de Paris, 2016), ainsi que celles en préparation de Marie Veltz au LATTS (UMR 8134) et de Félix Talvard au CSI (UMR 9217).

4. Sur ce point, la démarche s'inscrit dans le prolongement de notre thèse de doctorat (Stève Bernardin, La fabrique privée d'un problème public. La sécurité routière entre industriels et assureurs aux Etats-Unis (années 1920 à 2000), Université Paris I PanthéonSorbonne, 2014).

5. Nous tenons ainsi à contribuer à un programme de recherches collectives plus large visant à l'analyse des formes de mobilisation politique des élites économiques, à la suite notamment de Michel Offerlé, Sociologie des organisations patronales, Paris, La Découverte, 2009.

6. Madeleine Akrich, Stève Bernardin, Brice Laurent, David Pontille, Félix Talvard et alii, Experimenting in the City, Innovation and Controversies: The example of San Francisco and the Bay Area, Rapport de recherche du Centre de Sociologie de l'Innovation, 2016, 62 p.

7. L'ISSIP compte un millier de membres pour un budget annuel d'environ 250000 dollars. Elle propose des services distincts en fonction du montant de 
la cotisation des adhérents (de la gratuité à 20000 dollars par an), allant de l'accès à des rencontres professionnelles en cercles restreints à la délivrance de certificats d'aptitude, par exemple.

8. Sauf contre-indication de notre part, les citations sans référence renvoient aux prises de notes consignées dans notre carnet de terrain personnel.

9. P. K. Agarwal fait ici référence à la « TechHire Initiative » soutenue par la Présidence des États-Unis en 2015, ayant permis le déblocage de fonds à hauteur de 1,2 million de dollars à San José.

10. Michael Storper, Thomas Kemeny, Naji P. Makarem and Taner Osman, The Rise and Fall of Urban Economies. Lessons from San Francisco and Los Angeles, Stanford, Stanford University Press, 2015. Sur le cas spécifique de la Silicon Valley, en comparaison des technopôles français, voir Cynthia Ghorra-Gobin, « De l'alliance de la ville et de la haute technologie : les enseignements de la Silicon Valley ", in Espace géographique, tome 21, n 2, 1992, 109-118.

11. Entretien avec Jim Spohrer, directeur des programmes universitaires de la compagnie IBM, le 10 février 2016 dans la Silicon Valley.

12. Eliot Freidson, Professional Powers, Chicago, Chicago University Press, 1986.

13. Entretien avec Jim Spohrer, directeur des programmes universitaires de la compagnie IBM, le 10 février 2016.

14. Entretien avec Philippe Sajhau, vice-président d'IBM France en charge de l'initiative « Smarter cities », le 22 mai 2017 à Paris (par téléphone).

15. Donald McNeill, « Global firms and smart technologies : IBM and the reduction of cities », in Transactions of the Institute of British Geographers, vol. 40, issue 4, 2015, 562-574, p. 566.

16. Alan Wiig, « IBM's smart city as techno-utopian policy mobility », in City, vol. 19, issue 2-3, 2015, 258-273.
17. Entretien avec Peter Williams, responsable technique ( chief technological officer ») du programme « Big Green Innovations » de la compagnie IBM à San Francisco, le 19 février 2016 à San Francisco (par téléphone).

18. Entretien avec Krista Canellakis, adjointe au responsable de l'innovation (« deputy innovation officer ») de la ville de San Francisco, le 19 février 2016 à San Francisco.

19. Pour une analyse des expérimentations initiées pour tenter de répondre au problème diagnostiqué à San Francisco, voir Brice Laurent et Félix Talvard, « Real-time democracy. Imagining the city of permanent innovation ». i3 Working Papers Series, 17-CSI01, juillet 2017.

20. Alan Wiig, " The empty rhetoric of the smart city : from digital inclusion to economic promotion in Philadelphia », in Urban Geography, vol. 37, issue 4, 2016, 535-553.

21. Entretien avec Jim Spohrer, directeur des programmes universitaires de la compagnie IBM, le 10 février 2016.

22. Peter Williams, «The Internal Politics of Smarter Cities » (texte en ligne sur la page LinkedIn de l'auteur, consulté le 18 février 2016).

23. Sur le thème de la démonstration publique chez les spécialistes de la Silicon Valley, voir Claude Rosental, Les capitalistes de la science. Enquête sur les démonstrateurs de la Silicon Valley et de la NASA, Paris, CNRS Éditions, 2007.

24. Entretien avec Peter Williams, responsable technique du programme « Big Green Innovations » de la compagnie IBM à San Francisco, le 19 février 2016.

25. Entretien avec Jim Spohrer, directeur des programmes universitaires de la compagnie IBM, le 10 février 2016.

26. Idem.

27. Sur les pratiques philanthropiques des entrepre- 
neurs de la Silicon Valley, voir Marc Abélès, Les nouveaux riches. Un ethnologue dans la Silicon Valley, Paris, Odile Jacob, 2002.

28. Entretien avec Peter Williams, responsable technique du programme « Big Green Innovations » de la compagnie IBM à San Francisco, le 19 février 2016.

29. Propos de Philippe Sajhau, vice-président d'IBM France, rapporté dans les actes du $2^{\mathrm{e}}$ rendez-vous annuels d la «Cité des smart cities » organisé le 29 septembre 2015 : Smart cities : l'innovation au cœur de l'action publique?, Boulogne-Billancourt, BergerLevrault, 2016, p. 31.

30. François Ménard, «Penser la ville intelligente telle qu'elle est, la réguler telle qu'elle pourrait être », in Urbanisme (à paraître).

31. Entretien avec Philippe Sajhau, vice-président d'IBM France en charge de l'initiative « Smarter cities », le 22 mai 2017.

32. Entretien avec Peter Williams, responsable technique du programme « Big Green Innovations » de la compagnie IBM à San Francisco, le 19 février 2016.

33. Pour un point critique sur ce thème, voir Julien Talpin, « Légitimer les inégalités. De la démocratie participative en Amérique », in Participations, 1, 14, 2016, 265-282.

34. Entretien avec Krista Canellakis, adjointe au responsable de l'innovation de la ville de San Francisco, le 19 février 2016.

\section{$R \cdot E ́ \cdot S \cdot U \cdot M \cdot E ́$}

Écologie, rentable et sûre : la « ville intelligente » apparaît aujourd'hui comme une panacée. Ses promoteurs les plus enthousiastes en font une évidence : les technologies numériques constituent un remède idéal face aux problèmes présents et futurs des villes contemporaines. Leurs détracteurs dénoncent un discours technocratique, porté par des élites économiques ne tenant pas compte des réalités politiques et sociales. Le présent article vise à prendre au sérieux les deux perspectives, en interrogeant les conditions de production du discours des industriels du secteur numérique. Quelle en est la genèse économique et sociale ? Qui le porte concrètement, et comment? Des hypothèses fortes se dessinent avec une première enquête exploratoire menée au cœur de la Silicon Valley, en Californie. Elles invitent à ne pas céder trop rapidement aux facilités de la dénonciation technocratique, pour souligner un travail plus subtil de définition et de légitimation politique et sociale des problèmes publics auxquels est censé répondre l'avènement de la ville intelligente. À partir de l'ethnographie d'une conférence de professionnels du secteur, l'analyse témoigne plus précisément des contraintes autant que des ressources propres aux industriels en venant à porter leurs demandes en public. Elle amène finalement à interroger l'hypothèse d'une conformation de leurs porte-parole à un jeu politique dont ils cherchent aujourd'hui à maîtriser toutes les règles.

\footnotetext{
Abstract

To their promoters, "smart cities » are clean, safe, and sober. They come as an obvious remedy for some of the most critical problems of the cities worldwide. Their opponents do not share such optimism. They do not want of a technocratic discourse held by economic
} 
elites. To them, digital devices and sensors won't solve any political or social problems in the cities. This paper calls for a detailed analysis of both approaches. More precisely, it is an invitation to analyze the core sources of the current discourse on smart cities. Under which circumstances was it created? Who are its main promoters? How do they push now for their own agenda? The hypotheses presented here were developed after a series of interviews and ethnographical research in California, in February 2016. They shed light on a process through which social issues became a priority for some key actors of the digital economy. It led to a profound redefinition of the technocratic ideal of the smart city, to overcome some initial resistances expressed by local bureaucrats. The analysis not only puts the emphasis on the institutional constraints faced by the economic actors of the digital industry. It also underlines the key resources they now try to use to conform to what they perceive as the rules of the political field. 
